Figure 1. Lumbar Spine T-score and FRAX risk for OP

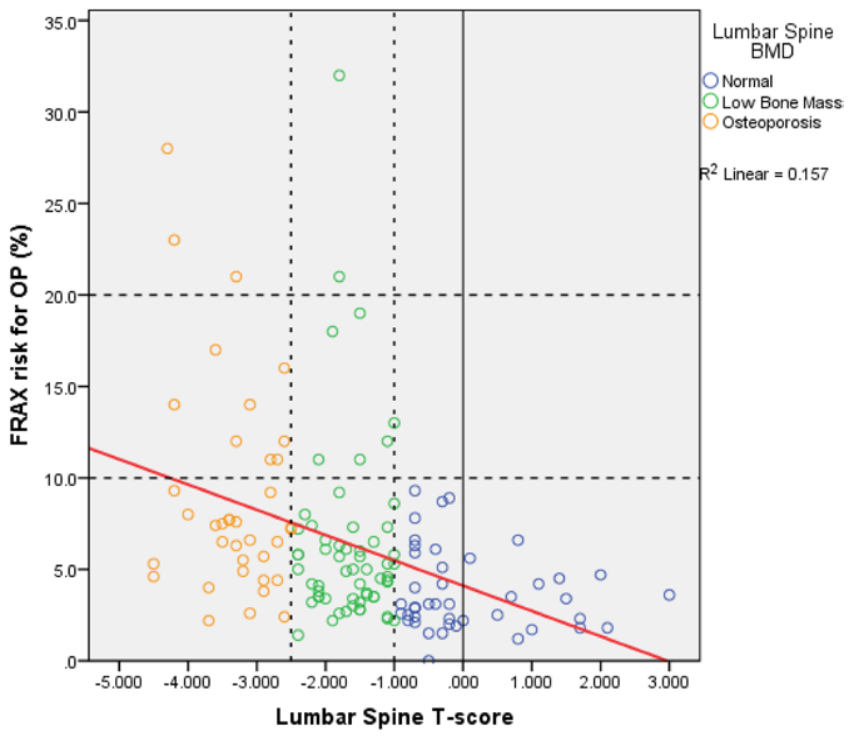

Figure 2. Femoral neck BMD and FRAX risk for Hip

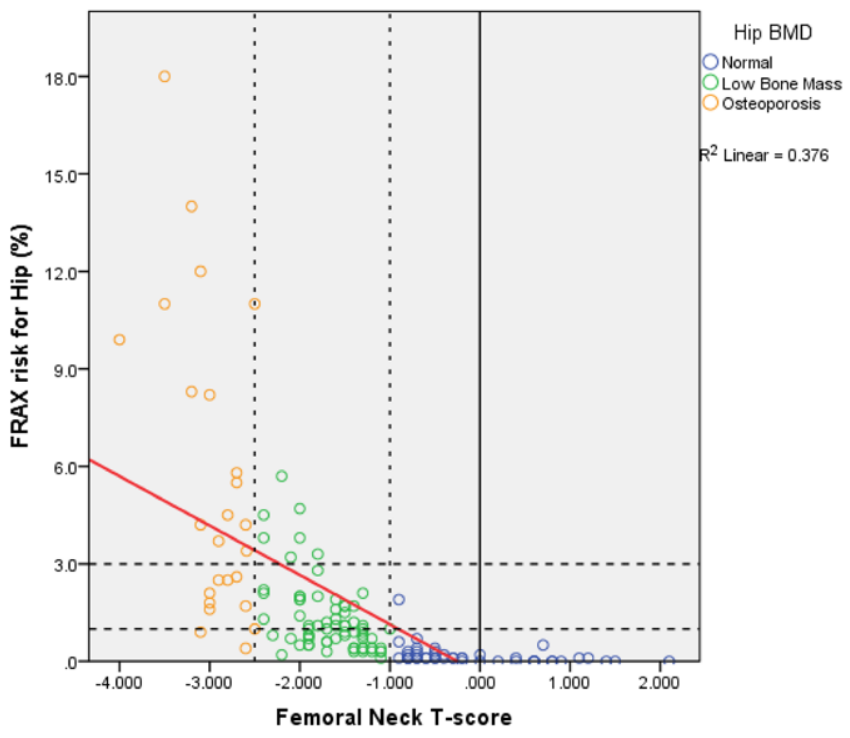

Disclosure of Interests: None declared

DOI: 10.1136/annrheumdis-2020-eular.5060

\section{SAT0467 \\ LOW SERUM OSTEOCALCIN LEVELS ARE ASSOCIATED WITH THE PRESENCE OF DIABETES MELLITUS IN GLUCOCORTICOID TREATED PATIENTS.}

$\underline{H}_{\text {H. Florez }}{ }^{1}$, J. Hernández-Rodríguez ${ }^{2}$, J. L. Carrasco ${ }^{3}$, S. Prieto-González ${ }^{2}$, X. Filella ${ }^{4}$, A. Monegal ${ }^{1}$, N. Guañabens ${ }^{1}$, P. Peris ${ }^{1} .{ }^{1}$ Hospital Clinic, University of Barcelona, Metabolic Bone Diseases Unit. Department of Rheumatology, Barcelona, Spain; ${ }^{2}$ Hospital Clinic, University of Barcelona, Vasculitis Research Unit, Department of Autoimmune Diseases, Barcelona, Spain; ${ }^{3}$ Hospital Clinic, University of Barcelona, Biostatistics, Department of Basic Clinical Practice, Barcelona, Spain; ${ }^{4}$ Hospital Clinic, Biochemistry and Molecular Genetics Department, Barcelona, Spain

Background: Increasing evidence indicates that osteocalcin $(\mathrm{OC})$ is involved in the regulation of glucose homeostasis. Glucocorticoid (GC) treatment is associated with impaired osteoblast function and decreased OC levels and also with the development of CG-induced diabetes mellitus (GIDM). However, whether decreased OC levels in GC-treated subjects contribute to GIDM is not well known.

Objectives: To analyse whether OC levels in GC-treated patients are associated with the presence of GIDM.
Methods: 127 patients (aged 62 \pm 18 years, 63\% women) on GC treatment for autoimmune diseases ( $\geq 5 \mathrm{mg} /$ day, $>3$ months) were included. Clinical and anthropometric data were analysed, including the GC dose and treatment duration, presence of GIDM, fragility fractures, densitometric osteoporosis and bone formation (OC, bone alkaline phosphatase [BAP], PINP) and resorption markers (urinary NTX, serum CTX). The cut-offs of each bone marker for the presence of GIDM were estimated and optimized with the Youden index and included in the logistic regression analysis (adjusted for BMI, age and GC doses).

Results: $17.3 \%$ of patients presented GIDM. Diabetic subjects were older $(70.5 \pm 12.2$ vs. $59.6 \pm 18.4, \mathrm{p}=0.001)$ and had a higher $\mathrm{BMI}$ than non-diabetics $(30 \pm 5.2$ vs. $26 \pm 4.2, p=0.002)$. No differences were observed in $G C$ dose or duration or in the presence of vertebral fractures. Diabetics showed lower levels of OC $(7.57 \pm 1.01$ vs. $11.56 \pm 1 ; p<0.001)$, PINP $(21.48 \pm 1.01$ vs. $28.39 \pm 1 ; p=0.0048), N T x$ $(24.91 \pm 1.01$ vs. $31.7 \pm 1 ; p=0.036)$ and CTX $(0.2 \pm 1.01$ vs. $0.3 \pm 1 ; p=0.0016)$ with similar BAP values. The best discriminating cut-offs for GIDM presence were: $<9.25 \mathrm{ng} / \mathrm{mL}$ for OC, $<24 \mathrm{ng} / \mathrm{mL}$ for PINP, $<27.5 \mathrm{nMol} / \mathrm{mM}$ for NTX and $<0.25 \mathrm{ng} /$ $\mathrm{mL}$ for CTX. On multivariate analysis $\mathrm{OC}(<9.25)$ was the only marker related to the presence of GIDM (OR 6.1; CI95\% 1.87-19.89; $p=0.001$ )

Conclusion: Decreased OC levels in GC-treated patients are associated with an increased risk of GIDM, a finding that was not observed with other bone turnover markers, further confirming the involvement of $\mathrm{OC}$ in the glucose homeostasis regulation in this entity.

Disclosure of Interests: None declared

DOI: 10.1136/annrheumdis-2020-eular.1519

\section{SAT0468 \\ EFFECTS OF ANDROGEN DEPRIVATION THERAPY ON BONE QUALITY (TBS) IN PATIENTS WITH PROSTATE CANCER}

S. Garcia-Cirera ${ }^{1}$, E. Casado ${ }^{1}$, J. Muñoz ${ }^{2}$, L. Del Río ${ }^{3}$, M. Arévalo ${ }^{1}$, M. Rusiñol ${ }^{1}$, N. Navarro ${ }^{1}$, V. Parejo ${ }^{2}$, J. Gratacos-Masmitja ${ }^{1}{ }^{1}{ }^{2}$ University Hospital Parc Tauli (UAB), Rheumatology, Sabadell, Spain; ${ }^{2}$ University Hospital Parc Taulí (UAB), Urology, Sabadell, Spain; ${ }^{3}$ CETIR Medical Center, Barcelona, Spain

Background: Androgen deprivation therapy (ADT), by inducing severe hypogonadism, leads to a loss of bone mineral density (BMD) and an increased risk of fragility fractures after 6 months of treatment in men with prostate cancer ${ }^{1}$. However, its effect on bone quality has not been described.

Objectives: To evaluate the changes on bone microarchitecture (bone quality) assessed by TBS (trabecular Bone Score) in male patients with prostate cancer after one year of treatment with ADT.

Methods: All patients diagnosed with prostate cancer candidates for long-term ADT admitted to Urology department of Hospital Universitari Parc Tauli (reference population of 450,000 inhabitants) between April 2017 and December 2019 were included. Patients who received chemotherapy, previous hormonal therapy or specific treatment for osteoporosis in the last year or those who had a very impaired functional capacity (Barthel index $<30$ ) were excluded.

Demographic, clinical and analytical data (testosterone, calcium, phosphorous, alkaline phosphatase, 25-hidroxyvitamin D, PTH) were collected in all patients. A bone densitometry (GE-Lunar Prodigy) including the measurement of lumbar spine TBS (L1-L4) using Medimaps Software was performed at baseline and at 12 months of treatment with ADT.

Results: 78 patients were included. Mean age $77,9 \pm 8,3$ years. The median Gleason score was $7,88 \pm 1,05$. 3 patients had previous fragility fracture (one sacral fracture, one fibula and one multiple vertebral fracture). Baseline analytical values in patients were the following: testosterone $11,6 \pm 74,9 \mathrm{nmol} / \mathrm{L}$.; 25 -hidroxyvitamin D 20,8 $\pm 10,4 \mathrm{ng} / \mathrm{ml}$; PTH $51,8 \pm 23,0 \mathrm{pg} / \mathrm{ml}$; CTX 0,58 $\pm 0,66$. The daily calcium intake was $573 \pm 207 \mathrm{mg} /$ day.

According to BMD, 17 patients $(21,8 \%)$ had osteoporosis before starting ADT, with the following average T-score values: lumbar spine $+0,15 \pm 1,85$, femora neck $-1,75 \pm 1,00$, and total hip $-1,19 \pm 1,16$. Mean baseline TBS value of the entire cohort was $1,279 \pm 0,122$. $30,5 \%$ of the patients showed very degraded microarchitecture (TBS $<1,230$ ), $28,8 \%$ had partially degraded microarchitecture (TBS $1,230-1,310$ ) and in $40,7 \%$ showed normal microarchitecture (TBS $>1,310$ ). After one year of ADT treatment, TBS mildly worsened in this cohort, with a median value of $1,256 \pm 0,131$ ( $p=N S$ ). However up to $43 \%$ of patients reached highly degraded microarchitecture, $27 \%$ partially degraded and only $29,5 \%$ had a normal TBS $(p=N S)$.

Conclusion: Most patients with prostate cancer have an altered bone quality before starting ADT. After 12 months of treatment, the percentage of patients with highly degraded bone microarchitecture increases, although not significantly. More studies are needed to confirm this trend and to evaluate if these patients present more long-term fractures.

References:

[1] Lee R, et al. Bone 2011; 48 (1): 88-95

Disclosure of Interests: Silvia Garcia-Cirera: None declared, Enrique Casado Speakers bureau: UCB, Lilly, Amgen, Theramex, Gebro, Gedeon-Richter, Stada, 